\title{
Evidence for cost-effectiveness of lifestyle primary preventions for cardiovascular disease in the Asia-Pacific Region: a systematic review
}

\author{
Lainie Sutton ${ }^{1}$, Anup Karan ${ }^{2,3^{*}}$ and Ajay Mahal ${ }^{1}$
}

\begin{abstract}
Background: Countries of the Asia Pacific region account for a major share of the global burden of disease due to cardiovascular disease (CVD) and this burden is rising over time. Modifiable behavioural risk factors for CVD are considered a key target for reduction in incidence but their effectiveness and cost-effectiveness tend to depend on country context. However, no systematic assessment of cost-effectiveness of interventions addressing behavioural risk factors in the region exists.
\end{abstract}

Methods: A systematic review of the published literature on cost-effectiveness of interventions targeting modifiable behavioural risk factors for CVD was undertaken. Inclusion criteria were (a) countries in Asia and the Pacific, (b) studies that had conducted economic evaluations of interventions (c) published papers in major economic and public health databases and (d) a comprehensive list of search words to identify appropriate articles. All authors independently examined the final list of articles relating to methodology and findings.

Results: Under our inclusion criteria a total of 28 studies, with baseline years ranging from 1990 to 2012, were included in the review, 19 conducted in high-income countries of the region. Reviewed studies assessed cost-effectiveness of interventions for tobacco control, alcohol reduction, salt intake control, physical activity and dietary interventions. The majority of cost-effectiveness analyses were simulation analyses mostly relying on developed country data, and only 6 studies used effectiveness data from RCTs in the region. Other than for Australia, no direct conclusions could be drawn about cost-effectiveness of interventions targeting behavioural risk factors due to the small number of studies, interventions that varied widely in design, and varied methods for measurement of costs associated with interventions.

Conclusions: Good quality cost-effectiveness information on interventions targeting behavioural interventions for the Asia-Pacific region remains a major gap in the literature.

Keywords: CVD, Systematic review, Cost effectiveness, Asia-pacific region

\section{Background}

In 2010, deaths due to cardiovascular disease (CVD) in the Asia-Pacific region accounted for $14 \%$ of all deaths and $48 \%$ of CVD-related deaths worldwide [1]. The share of CVD-related deaths in all-cause deaths in the Asia-Pacific region has also been rising over time - from $40 \%$ in 1990 to $48 \%$ in 2010 [1,2]. The most recent figures from the Institute for Health Metrics and Evaluation in 2010 also show

\footnotetext{
* Correspondence: anup.karan@iiphg.org

${ }^{2}$ Indian Institute of Public Health Gandhinagar (IIPHG), Sardar Patel Institute Campus, Thaltej, Ahmedabad 380 054, India

${ }^{3}$ Nuffield Department of Population Health, University of Oxford, Oxford, UK Full list of author information is available at the end of the article
}

that countries of the Asia-Pacific Region experienced 52\% of global disability-adjusted life years (DALYs) lost due to CVD (World Health Organization (WHO)) [2,3].

The growing incidence of CVD implies adverse economic consequences for countries in the region $[2,4]$. Thus, smoking in China was estimated to impose an economic burden of US $\$ 28.9$ billion in 2008, with medical treatment costs rising by $154 \%$ and indirect costs (such as foregone productivity) by $376 \%$ between 2000 and 2008 [5]. It has also been projected that in the absence of preventive action, CVD could cost Pakistan US\$31 billion and India US $\$ 237$ billion by 2015 in lost GDP [6]. A recent study [7] suggests 
that heart disease significantly increased out of pocket health spending, reduced labour force participation rate and increased indebtedness among Indian households.

In 2010, the vast majority of CVD-related deaths in the Asia-Pacific Region were due to hypertensive and ischemic heart disease, and stroke [1]. Risk factors for these conditions can be behavioural, metabolic, (hypertension, hyperglycaemia, abnormal serum lipid levels, or overweight/ obesity), psychological or genetic [8]. Much of the research on CVD risk factors comes from high income countries, but it has been shown that the various metabolic risk factors are also rising in the Asia Pacific Region [9]. Evidence from the Framingham Heart Study, the WHO MONICA Project and elsewhere has also shown that behavioural modification can influence metabolic risk factors for CVD [10-12]. Examples of behavioural changes include reduced intake of dietary salt, smoking cessation, trans-fat being replaced by polyunsaturated fat, and increased physical activity [8].

\section{Why it is important to do this review}

Although the causal linkages between primary prevention and CVD-related morbidity and mortality outcomes are well understood, the evidence on the feasibility and costeffectiveness of such primary prevention interventions is less clear in the Asia-Pacific region, especially in low- and middle-income countries. Studies conducted predominantly in high-income settings suggest that behavioural lifestyle interventions aimed at lowering morbidity and mortality due to CVD are cost-effective when implemented at the population level or when targeting high risk groups $[13,14]$. A recent WHO report also suggests addressing behavioural risk factors due to their cost-effectiveness, and their relative ease and speed of implementation $[15,16]$.

However, feasibility and cost-effectiveness tend to be context-specific and difficult to generalise across countries [14]. One study [17] modelled population-wide salt and tobacco reduction interventions for several countries and found large variations in government spending associated with implementation (China US\$22 per capita; Vietnam US\$7 per capita; and Philippines US\$14 per capita). To our knowledge no other review has examined the evidence for cost-effectiveness of various CVD lifestyle primary preventions in the Asia-Pacific Region, although a review from 2012 examined pharmaceutical primary preventive interventions in low- and middle-income countries of the region [18].

Although our focus is on low- and middle-income countries of the region, we included Australia, New Zealand and Japan for three reasons. First, there are migration links between these countries and the rest of the region: nearly two-thirds of all migrants to Australia were from the Asia-Pacific region in 2012-13 and lessons from the Australian experience may apply to specific country settings [19]. Second there are institutional linkages. For instance, the World Health Organisation's Asia Pacific Observatory on Health Systems and Policies which connects scientific and policy-making bodies throughout the region and acts as a conduit for promoting best-practice health programs throughout the region based on evidence sharing includes both Australia and New Zealand in its membership [20]. In this context, Australia's tobacco-related policy actions could be of great relevance in much of Asia, including China and India [21]. Finally, the epidemiological profile of the population in countries of the region will increasingly resemble its more developed counterparts in the region, reflecting rising incomes and convergence in dietary patterns towards western-style foods [22]. Thus interventions that have previously been considered in the high-income countries of the region may become relevant for their low- and middle-income counterparts in future years.

\section{Methods}

\section{Types of primary prevention interventions}

This review was restricted to primary prevention interventions among adults as long as they targeted at least one of the main behavioural risk factors for CVD. The interventions covered included (but were not restricted to) nutritional programs, physical activity programs (individual/ group/community-wide programs), food taxation, salt reduction programs, health promotion advertising, behavioural counselling (dietary, physical activity etc. versus standard care/no primary prevention).

\section{Types of studies}

Studies were considered for evaluation if they were in the form of [i] economic evaluation studies (i.e. cost-effectiveness analyses, cost-utility analyses, cost-benefit analyses) of primary lifestyle/behavioural interventions for CVD using case-control, cohort, cross-sectional or randomised controlled trial methods; [ii] simulation studies estimating economic effectiveness for lifestyle/behavioural primary prevention interventions for CVD; [iii] studies published in English; [iv] studies that were limited to adult populations in Asia and the Pacific.

Studies were excluded if they [i] were in the form of letters, abstracts, comments, case reports, editorials, descriptive studies, ecological studies and conference papers; [ii] involved non-human subjects; [iii] were conducted outside of the Asia-Pacific Region; and [iv] did not include information on outcomes (e.g., pure costing studies), or did not include information on intervention costs (only gross economic benefits were estimated).

\section{Search strategy}

During October 2013 we searched major information sources such as the Cochrane database, Pubmed, Scopus, Database of Promoting Health Effectiveness reviews and 
the website of the WHO (for the full list of sources and search strategy see Additional file 1).

\section{Study selection}

Search results produced titles and abstract for all studies, which were then assessed against our eligibility criteria to determine inclusion for full text review. If an article was rejected, the reason was recorded; if a study was earmarked for inclusion, it was reviewed in its entirety according to an extended standardised eligibility checklist (Additional file 2).

\section{Data collection process and data items}

Pre-determined data was extracted from each of the studies selected for inclusion in the review using a tested data extraction form (Additional file 3). This data included the year of publication, setting (country and within-country location), target population, study type, economic modelling method, intervention type, health implications, uncertainty considerations made (i.e. sensitivity analysis, discounting), economic outcomes and costs and the perspective used (health sector, societal etc.).

\section{Risk of bias in individual studies (quality review)}

Full text articles were appraised for quality using the criteria for economic studies' evaluation in Evers et al. [23]. Studies were ranked, with a 'low-risk' study $(++)$ deemed to have the least risk of bias and considered to be a rigorous economic study (Additional file 4). The quality criteria took into account the design of the study in terms of the participants, the primary prevention and control alternative; the frequency, duration and intensity of any intervention; measurable outcomes; whether any conflicts of interest were present; and whether ethical approval was obtained. The study design was assessed in terms of the type of evaluation, the basis of this evaluation (e.g., randomised controlled trial, cross-sectional study, simulation modelling, etc.) and the perspective of the study, where a societal perspective is considered the default position. If another perspective was taken an explanation would have to be provided as to why certain costs or effects were not considered or included. If a study period exceeded one year, discounting of future costs would have to be demonstrated. The method for evaluation should have been clearly stated and all relevant outcomes shown, with sensitivity analysis. Studies were ranked according to the NICE scale from ++ to + to - from lowest to highest risk of bias [24].

\section{Synthesis of studies}

Studies were initially classified by whether they included cost-effectiveness analyses (CEA), where cost is relative to some utility measure (e.g. DALY, QALY, LYS) or another economic evaluation approach (e.g., cost-benefit analysis). For CEA, an assessment of cost-effectiveness was then made for each study or primary prevention intervention within each study. A study was considered very costeffective if cost per DALY saved was less than GDP per capita, cost-effective if cost per DALY was between 1 to 3 times GDP per capita and not cost-effective otherwise [25]. GDP per capita for the year in question was obtained from the World Bank Development Indicators Database [26].

\section{Results}

\section{Study selection and characteristics}

The search yielded 16,725 results, of which 87 full texts were retrieved for further assessment. Subsequently, 28 were deemed suitable to be included in the qualitative review using our selection criteria. Primarily studies were excluded due to the research design being incompatible with our criteria and prevention interventions not being lifestyle or behavioural (Figure 1).

The studies retrieved for review predominantly used the period 2000-2012 as baseline years, with 4 additional studies [25,27-29] using 1990 to 1997 as baseline (the studies are summarized in Table 1).

Almost two-thirds of the studies were located within high-income countries of the region (19/28), with 16 in Australia. A large number (22/28) were simulation analyses, while 6 cost-effectiveness assessments were embedded within randomised controlled trial prevention studies $[25,27,28,40,45,46]$. Only 2 studies $[28,46]$ did not include some type of sensitivity analysis for their findings. 18 of the studies were population-based and most studies (23/ 28) discussed the generalizability of their findings for other populations. Many studies took a government perspective (including costs of the intervention and healthcare cost offsets), with 4 taking a perspective that included the associated household economic burden [33,34,38,45]. 19 of the 28 studies included DALYs or QALYs as outcomes, based either on CVD outcomes alone, or on CVD outcomes plus cancers and diabetes (and COPD in some cases), for costeffectiveness analyses. Outcomes in other studies included indicators of changes in risk factors for CVD (e.g., weight reduction, number of smokers quitting, physical activity and systolic BP), and deaths averted.

Given the focus of primary prevention interventions on lifestyle behaviours, it was expected that authors take into account programme compliance rates of participants and their decay over time [54]. Many studies considered this aspect in the analysis, yet $7 / 28$ did not provide any estimate of the effect of decay $[28,37,45-48,52]$. Finally, with a few exceptions $[28,29,43,49]$ that did not itemize costs, most included studies used appropriate costing for interventions and other peripheries.

\section{Risk of bias across studies}

Table 2 reports our findings on the risk of bias across the included studies. 


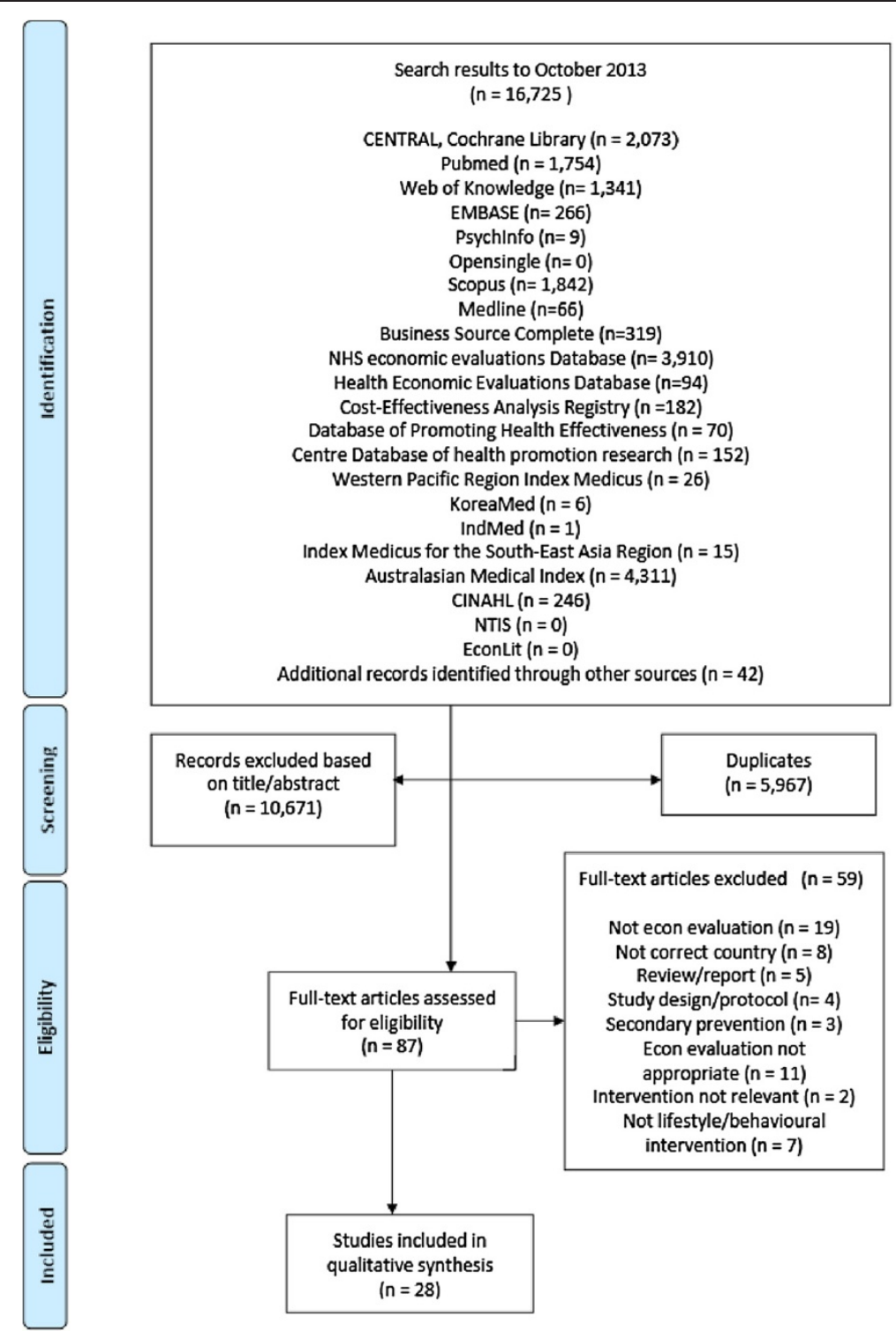

Figure 1 Flow diagram of studies in the Asia Pacific Region investigating cost-effectiveness of lifestyle or behavioural interventions for cardiovascular disease primary prevention.

Only one study [53] used industry funding but it was specified that there was no conflict of interest with the funding body. 8 of the 28 studies had a potential for conflict of interest based on the lack of a disclaimer, a vague disclaimer or the possibility of stakeholder involvement in the analyses $[25,27,38-40,46,51,52]$. We also compared estimates of primary prevention effectiveness when there was some conflict of interest in the study with estimates of primary prevention effectiveness when studies were deemed to have no conflict of interest. Studies which had some risk of conflict of interest demonstrated a positive effect of the intervention (cost-effective to very cost-effective outcome) about 70 percent of the time, whereas 80 percent of the studies deemed to be without a conflict of interest showed a positive effect of the intervention. Most studies' conclusions followed the results provided, with a few exceptions providing incomplete or selective conclusions relative to the results observed. Overall we deemed $7 /$ 28 studies to be at low risk of bias $[27,28,36,37,39,41,48]$ (with all of these studies conducted in Australia), 12/28 studies to be high risk [31-33,40,42,43,47,49-53] and the remainder $(9 / 28)$ as having a moderate risk of bias.

\section{Evidence for cost-effectiveness of primary prevention}

Many studies evaluated multiple primary prevention interventions, and some targeted multiple risk factors. For example Jafar et al. [45] assessed 3 interventions for hypertensive patients: a home-based health education 
Table 1 Study characteristics: location, study design and economic perspective

\begin{tabular}{|c|c|c|c|c|c|c|c|}
\hline Study & $\begin{array}{l}\text { Country/baseline } \\
\text { year }\end{array}$ & Intervention length & Follow-up & Study population & Key intervention components & Control & $\begin{array}{l}\text { Perspective } \\
\text { (e.g. health } \\
\text { sector) }\end{array}$ \\
\hline Amarasinghe [30] & Australia, 2006 & 1 Year & 1 Year & $\begin{array}{l}18 \text { yrs + in Western } \\
\text { Australia, male and } \\
\text { female, CVD risk } \\
\text { level unknown }\end{array}$ & $\begin{array}{l}\text { GP advice for sufficient physical } \\
\text { activity ( } 150+\text { minutes/week) } \\
\text { upon random presentation at } \\
\text { clinic ( } 6 \text { visits) }\end{array}$ & $\begin{array}{l}\text { No GP advice on } \\
\text { physical activity }\end{array}$ & Health Sector \\
\hline Cecchini [31] & China, India, 2008 & $\begin{array}{l}\text { Lifetime of population } \\
\text { at baseline or up to } \\
100 \text { yrs }\end{array}$ & Lifetime & Whole population & $\begin{array}{l}\text { Int1 Worksite health promotion; Int2 } \\
\text { Compulsory food labelling; Int3 Mass } \\
\text { media health promotion campaigns; } \\
\text { Int4 Fiscal measures affecting fruit } \\
\text { and vegetable and food high in fat }\end{array}$ & No intervention & Health sector \\
\hline Cobiac [32] & Australia, 2003 & $\begin{array}{l}\text { Lifetime of population } \\
\text { at baseline or up to } \\
100 \text { yrs }\end{array}$ & Lifetime & $\begin{array}{l}\text { Whole population } \\
\text { (15+ yrs) }\end{array}$ & $\begin{array}{l}\text { Int1 Volumetric taxation; Int2 } \\
\text { Advertising bans; Int3 Licensing } \\
\text { controls on operating hours; Int4 } \\
\text { Brief intervention by GP; Int } 5 \text { Brief } \\
\text { intervention by GP with GP } \\
\text { telemarketing and support; Int6 } \\
\text { Residential treatment; Int7 Increase } \\
\text { legal age; Int8 Drink driving campaigns. }\end{array}$ & $\begin{array}{l}\text { Current best } \\
\text { practice/'do nothing' } \\
\text { (random breath } \\
\text { testing present) }\end{array}$ & Health sector \\
\hline Cobiac [33] & Australia, 2003 & $\begin{array}{l}\text { Lifetime of population } \\
\text { at baseline }\end{array}$ & 6 Months & $\begin{array}{l}\text { Whole population } \\
\text { (adult, BMl } \geq 25 \mathrm{~kg} / \mathrm{m}^{2} \text {, } \\
\text { don't eat at least } 7 \\
\text { serves of fruit and } \\
\text { vegetables/day, don't } \\
\text { get } 30+\text { minutes } \\
\text { moderate exercise at } \\
\text { least } 5 x / \text { week) }\end{array}$ & $\begin{array}{l}\text { Int1 Lighten up (group counselling } \\
\text { for changing physical activity and } \\
\text { nutrition patterns); Int2 Weight } \\
\text { watchers (low-calorie diet and } \\
\text { physical activity advice) }\end{array}$ & No intervention & Health sector \\
\hline Cobiac [34] & Australia, 2003 & $\begin{array}{l}\text { Lifetime of population } \\
\text { at baseline }\end{array}$ & Lifetime & $\begin{array}{l}\text { Whole population } \\
\text { (15+ yrs at baseline) }\end{array}$ & $\begin{array}{l}\text { Int1 Pedometers; Int2 Mass media } \\
\text { campaign; Int3 Internet advertising; } \\
\text { Int4 GP physical activity prescription } \\
\text { program; Int5 Travel smart program } \\
\text { to encourage use of active transport; } \\
\text { Int6 GP referral to exercise physiologist. }\end{array}$ & Current practice & $\begin{array}{l}\text { Societal and } \\
\text { health sectors }\end{array}$ \\
\hline Cobiac [35] & Australia, 2003 & $\begin{array}{l}\text { Lifetime of population } \\
\text { at baseline }\end{array}$ & Lifetime & $\begin{array}{l}\text { Whole population } \\
\text { (30+yrs at baseline) }\end{array}$ & $\begin{array}{l}\text { Int1 Govt. incentives for moderate } \\
\text { reduction in salt in processed foods } \\
\text { by manufacturers and product } \\
\text { labelling (voluntary); Int2 Govt. } \\
\text { mandate to moderate salt limits in } \\
\text { processed foods; Int3 Dietary advice } \\
\text { for those at increased risk of CVD; } \\
\text { Int4 Dietary advice for those at high } \\
\text { risk of CVD }\end{array}$ & No intervention & Health sector \\
\hline Cobiac [36] & Australia, 2003 & $\begin{array}{l}\text { Lifetime of population } \\
\text { at baseline }\end{array}$ & Lifetime & Whole adult population & $\begin{array}{l}\text { Int1 Community-based events, } \\
\text { sponsorship, promotion; Int2 } \\
\text { Information mail-out (multiple } \\
\text { re-tailored); Int3 Information } \\
\text { mail-out (multiple tailored); Int4 }\end{array}$ & No intervention & Health sector \\
\hline
\end{tabular}


Table 1 Study characteristics: location, study design and economic perspective (Continued)

\begin{tabular}{|c|c|c|c|c|c|c|c|}
\hline & & & & & $\begin{array}{l}\text { Information mail-out (tailored); } \\
\text { Int5 Individual and group dietary } \\
\text { counselling; Int6 Individual dietary } \\
\text { counselling; Int7 Telephone counselling } \\
\text { and information mail-out }\end{array}$ & & \\
\hline Cobiac [37] & Australia, 2008 & $\begin{array}{l}\text { Lifetime of population } \\
\text { at baseline }\end{array}$ & Lifetime & $\begin{array}{l}\text { Whole population } \\
\text { (35-84 yrs at baseline, } \\
\text { never experienced } \\
\text { heart disease or stroke, } \\
\text { all CVD risk levels) }\end{array}$ & $\begin{array}{l}\text { Community: Int1 Heart health program; } \\
\text { Int2 Mandatory reduction of salt in } \\
\text { manufacture of bread, cereals and } \\
\text { margarines. Individual: Int3 Dietary } \\
\text { advice from doctor or dietician; Int4 } \\
\text { Referral to more intensive lifestyle } \\
\text { program with specialised counselling } \\
\text { ( } \geq 15 \% \text { risk of CVD); Int5 Advice from } \\
\text { doctor to switch to phytosterol enriched } \\
\text { margarine ( } \geq 15 \% \text { risk of CVD) }\end{array}$ & $\begin{array}{l}\text { Current best practice } \\
\text { ('do nothing') }\end{array}$ & Health sector \\
\hline Dalziel [38] & Australia, 2003 & 6 Months -6 Years & $\begin{array}{l}1 \text { session } \\
-1 \text { Year }\end{array}$ & $\begin{array}{l}\text { Unclear - clinical trials } \\
\text { were in adults }\end{array}$ & 8 dietary interventions & No intervention & $\begin{array}{l}\text { Societal } \\
\text { perspective }\end{array}$ \\
\hline Dalziel [39] & New Zealand, 2000 & Lifetime (40 years) & 1 Year & $\begin{array}{l}\text { Adults } 40-79 \text { yrs, } \\
\text { M\&F, not getting } \\
2.5 \text { hrs physical } \\
\text { activity per week } \\
(\mathrm{n}=878)\end{array}$ & $\begin{array}{l}\text { Physical activity counselling program } \\
\text { (verbal advice and written exercise } \\
\text { program by GP or Nurse and telephone } \\
\text { exercise specialist follow-up) }\end{array}$ & Best practice & Health Sector \\
\hline Elley [40] & New Zealand, 2000 & 1 Year & 3 Months & $\begin{array}{l}\text { Adults } 40-79 \text { yrs, } \\
\text { M\&F, not getting } \\
2.5 \text { hrs physical } \\
\text { activity per week } \\
(\mathrm{n}=878)\end{array}$ & $\begin{array}{l}\text { Physical activity counselling program } \\
\text { (verbal advice and written exercise } \\
\text { program by GP or Nurse and telephone } \\
\text { exercise specialist follow-up) }\end{array}$ & Best practice & $\begin{array}{l}\text { Programme } \\
\text { funder } \\
\text { Perspective }\end{array}$ \\
\hline Forster [41] & Australia, 2003 & $\begin{array}{l}\text { Lifetime of population } \\
\text { at baseline or } 100 \text { yrs }\end{array}$ & 6-12 Months & $\begin{array}{l}\text { Adults } 20 \text { yrs + } \\
\text { overweight and } \\
\text { obese }\end{array}$ & $\begin{array}{l}\text { Int1:Hypertension diet with exercise; } \\
\text { Int2:Low-fat diet }\end{array}$ & No intervention & Health sector \\
\hline Ha [42] & Vietnam, 2007 & $\begin{array}{l}\text { Lifetime of population } \\
\text { at baseline or up to } \\
100 \text { yrs }\end{array}$ & 10 Years & $\begin{array}{l}\text { Adult population } \\
30+\text { years at baseline, } \\
\text { all risk levels }\end{array}$ & $\begin{array}{l}\text { Int1: Reduction in salt intake through } \\
\text { voluntary manufacturer limits, mass } \\
\text { media campaign; Int2:Mass media } \\
\text { campaign to reduce cholesterol; } \\
\text { Int3:Mass media campaign to reduce } \\
\text { tobacco; Int4:Interventions 1-3 combined }\end{array}$ & No intervention & Health sector \\
\hline Higashi [43] & Vietnam, 2006 & $\begin{array}{l}\text { Lifetime of population } \\
\text { at baseline }\end{array}$ & 1 Session & $\begin{array}{l}\text { Adult population } 15+y \\
\text { at baseline, stratified by } \\
\text { i) never smoker, ii) current } \\
\text { smoker, iii) ex-smoker }\end{array}$ & $\begin{array}{l}\text { Brief physician advice (GP or other } \\
\text { health professional) on tobacco } \\
\text { cessation ( } 1 \text { min screening and } 8 \text { min } \\
\text { advice session) }\end{array}$ & $\begin{array}{l}\text { Pharmaceutical } \\
\text { intervention (NRT } \\
\text { patch, NRT gum, } \\
\text { Bupropion, } \\
\text { Varenicline) }\end{array}$ & Health sector \\
\hline Higashi [44] & Vietnam, 2006 & $\begin{array}{l}\text { Lifetime of population } \\
\text { at baseline }\end{array}$ & 1-10 Years & $\begin{array}{l}\text { Adult population } 15+y r \\
\text { at baseline, all risk levels }\end{array}$ & $\begin{array}{l}\text { Int1: Excise tax increase (55-65\%); Int2: } \\
\text { Excise tax increase (55-75\%); Int3: Excise } \\
\text { tax increase (55-85\%); Int4: Graphic } \\
\text { warning labels on cigarette packs; Int5: } \\
\text { Mass media campaigns; Int6: Smoking }\end{array}$ & No intervention & $\begin{array}{l}\text { Government } \\
\text { perspective } \\
\text { (including initial } \\
\text { investment in } \\
\text { interventions) }\end{array}$ \\
\hline
\end{tabular}


Table 1 Study characteristics: location, study design and economic perspective (Continued)

\begin{tabular}{|c|c|c|c|c|c|c|c|}
\hline & & & & & $\begin{array}{l}\text { bans in public; Int7: Smoking bans in } \\
\text { workplace. All enforced for } 10 \text { years. }\end{array}$ & & \\
\hline Jafar [45] & Pakistan, 2007 & 2 years & 2 Years & $\begin{array}{l}\text { Adult male and females } \\
40+\text { years, hypertensive }\end{array}$ & $\begin{array}{l}\text { Home health education (HHE) and } \\
\text { training of GPs on BP control - Int1 } \\
: \mathrm{HHE}+\text { trained GP; Int } 2: \mathrm{HHE} \text {; Int } 3: \\
\text { Trained GP }\end{array}$ & Current practice & Societal \\
\hline Joo [46] & South Korea, 2007 & 12 weeks & 12 Weeks & $\begin{array}{l}\text { Adults } 20-64 \mathrm{yrs}, \mathrm{BMI} \geq \\
25 \mathrm{~kg} / \mathrm{m}^{2}, \text { waist } \\
\text { circumference }>90 \mathrm{~cm} \\
\text { men, } 85 \mathrm{~cm} \text { women, } \\
30 \text { min exercise } 4 \\
\text { times/week }\end{array}$ & $\begin{array}{l}\text { Protein-rich oriental diet and either } \\
\text { of:- Int1: Public health centre } \\
\text { behavioural program; Int } 2 \text { : Remote } \\
\text { behavioural program (internet, SMS) }\end{array}$ & None & $\begin{array}{l}\text { Program funder } \\
\text { perspective }\end{array}$ \\
\hline Murray [47] & $\begin{array}{l}\text { South East Asia Region } \\
\text { (Indonesia, Sri Lanka, } \\
\text { Thailand, Bangladesh, } \\
\text { Bhutan, Dem Peop. Rep. } \\
\text { Korea, India, Maldives, } \\
\text { Myanmar, Nepal), } 2000\end{array}$ & $\begin{array}{l}\text { Lifetime of population } \\
\text { at baseline or } 100 \\
\text { years }\end{array}$ & Lifetime & Whole population & $\begin{array}{l}\text { Population interventions for BP and } \\
\text { cholesterol control: Int1:Voluntary } \\
\text { agreements on salt content with } \\
\text { manufacturers; Int2:Legislated salt } \\
\text { limits in manufactured food; Int3:Mass } \\
\text { media campaign; Int4 : Int2 + Int3 }\end{array}$ & Current practice & $\begin{array}{l}\text { Government } \\
\text { (implementation } \\
\text { and health sector } \\
\text { costs) }\end{array}$ \\
\hline Navarro [48] & Australia, 2005 & 1 year & 1 Session & $\begin{array}{l}\text { Adults } 18+\text { yrs at } \\
\text { baseline from } 10 \text { rural } \\
\text { communities in NSW, } \\
\text { stratified by drinking } \\
\text { behaviour }\end{array}$ & $\begin{array}{l}\text { GP screening and brief intervention } \\
\text { (1 session) }\end{array}$ & $\begin{array}{l}\text { Current best } \\
\text { practice }\end{array}$ & Health sector \\
\hline Oldenburg [25] & Australia, 1990 & 1 year & 1 Year & $\begin{array}{l}\text { Adult male and female } \\
\text { ambulance officers and } \\
\text { paramedics }\end{array}$ & $\begin{array}{l}\text { Int1:Health risk assessment (4x over } \\
12 \text { months); Int2 : Risk factor education } \\
\text { (4x over } 12 \text { months plus reading } \\
\text { material); Int } 3 \text { : Behavioural counselling } \\
\text { (risk factor education plus } 1 \text { session } \\
\text { behavioural counselling); Int 4:Behavioural } \\
\text { counselling plus financial incentives }\end{array}$ & No intervention & $\begin{array}{l}\text { Program funder } \\
\text { perspective }\end{array}$ \\
\hline Ortegon [49] & $\begin{array}{l}\text { South East Asia Region } \\
\text { (Bangladesh, Bhutan, } \\
\text { Dem. People's Republic } \\
\text { of Korea, India, Maldives, } \\
\text { Myanmar, Nepal), } 2005\end{array}$ & $\begin{array}{l}\text { Lifetime of population } \\
\text { at baseline or up to } \\
100 \text { yrs }\end{array}$ & 10 Years & $\begin{array}{l}\text { Whole population } \\
15 \text { yrst }\end{array}$ & $\begin{array}{l}\text { Interventions implemented for } 10 \text { years } \\
\text { Int1: Taxes on tobacco (current excise } \\
\text { taxation of } 40 \% \text { ); Int2:Raise taxes on } \\
\text { tobacco (increased excise taxation to } \\
60 \% \text { ); Int3:Enforce bans on tobacco } \\
\text { advertising; Int4:Clean indoor air in public } \\
\text { places through legislation and enforcement; } \\
\text { Int5:Warning labels on cigarette packs; } \\
\text { Int6:Brief advice to help quit; Int7:Counselling } \\
\text { to help quit; Int8: Voluntary reduction in } \\
\text { salt in industry (15\%); Int9: Legislated } \\
\text { reduction in salt in industry (30\%); Int10:Mass } \\
\text { media education on BMl and cholesterol }\end{array}$ & No Intervention & $\begin{array}{l}\text { Program funder } \\
\text { and health sector }\end{array}$ \\
\hline Pritchard [28] & Australia, 1992 & 1 year & 1 Year & $\begin{array}{l}25-65 \text { yr old men and } \\
\text { women with one or } \\
\text { more of: overweight, }\end{array}$ & $\begin{array}{l}6 \text { sessions of counselling on good nutrition } \\
\text { and exercise by: Int1: Doctor/dietician; Int2: } \\
\text { Dietician only }\end{array}$ & No counselling & Program funder \\
\hline
\end{tabular}


Table 1 Study characteristics: location, study design and economic perspective (Continued)

\begin{tabular}{|c|c|c|c|c|c|c|c|}
\hline & & & & $\begin{array}{l}\text { hypertension, type } 2 \\
\text { Diabetes mellitus }\end{array}$ & & & \\
\hline Ranson [29] & $\begin{array}{l}\text { East Asia \& Pacific, } \\
\text { South Asia, } 1995\end{array}$ & $\begin{array}{l}\text { Lifetime of participants } \\
\text { at baseline }\end{array}$ & Lifetime & Smokers $15+$ years & $\begin{array}{l}\text { Public policy control interventions: } \\
\text { Int1: } 10 \% \text { price increase; Int2 : Non-price } \\
\text { increase, non-pharmaceutical (e.g. mass } \\
\text { media) }\end{array}$ & 'Do nothing' & $\begin{array}{l}\text { Program funder } \\
\text { (public sector) }\end{array}$ \\
\hline Sacks [50] & Australia, 2003 & $\begin{array}{l}\text { Lifetime of participants } \\
\text { at baseline }\end{array}$ & Lifetime & $\begin{array}{l}\text { Whole population } \\
\geq 20 \text { years at baseline }\end{array}$ & $\begin{array}{l}\text { Int1: Traffic light labelling of food based } \\
\text { on nutritional content; Int2 }: \text { Junk food tax } \\
\text { (10\% rise in prices for consumers) }\end{array}$ & No intervention & $\begin{array}{l}\text { Health sector } \\
\text { (with some } \\
\text { industry costs } \\
\text { included) }\end{array}$ \\
\hline Salkeld [27] & Australia, 1990 & $\begin{array}{l}\text { Lifetime of participants } \\
\text { at baseline }\end{array}$ & 1 Year & $\begin{array}{l}\text { Male and female, } \\
\text { selected by GP for } \\
\text { at risk of CVD }\end{array}$ & $\begin{array}{l}\text { Int1:Video intervention for lifestyle } \\
\text { behaviours }(n=270) \text {; Int2:Video + } \\
\text { self-help booklet }(n=232)\end{array}$ & $\begin{array}{l}\text { Routine care } \\
(n=255)\end{array}$ & $\begin{array}{l}\text { Health sector } \\
\text { (govt.) }\end{array}$ \\
\hline Shearer [51] & Australia, 2003 & 6 months & 6 Months & $\begin{array}{l}\text { Whole adult population, } \\
\text { smokers }\end{array}$ & $\begin{array}{l}\text { Int1:Brief advice by health professional } \\
\text { ( } 2 \times 10 \text { min visits); Int2:Telephone } \\
\text { counselling ( } 4 \times 10 \text { min calls) }\end{array}$ & No intervention & $\begin{array}{l}\text { Program funder } \\
\text { (govt.) }\end{array}$ \\
\hline Snowdon [52] & Fiji, Tonga, 2006 & Lifetime & Lifetime & Whole population & $\begin{array}{l}\text { Policy changes around food price, } \\
\text { storage, manufacture, items available } \\
\text { for consumption }\end{array}$ & 'Do nothing' & $\begin{array}{l}\text { Govt. (cost offsets } \\
\text { excluded) }\end{array}$ \\
\hline Zomer [53] & Australia, 2012 & $\begin{array}{l}10 \text { years or death of } \\
\text { baseline population }\end{array}$ & 10 Years & $\begin{array}{l}10,000 \text { adults } \geq 25 \text { yrs } \\
\text { with hypertension and } \\
\text { metabolic syndrome, } \\
\text { no CVD history (based } \\
\text { on subsection of } \\
\text { AusDiab Study } \\
\text { participants) }\end{array}$ & $\begin{array}{l}\text { Daily consumption of dark chocolate } \\
(500-1000 \mathrm{mg} / \text { day })\end{array}$ & $\begin{array}{l}\text { No dark chocolate } \\
\text { consumption }\end{array}$ & Health sector \\
\hline
\end{tabular}


Table 2 Assessing risk of bias in studies included in the review

\begin{tabular}{|c|c|c|c|c|c|c|c|c|}
\hline Study & $\begin{array}{l}\text { Sensitivity analysis; discount rate } \\
\text { (per annum); perspective (e.g. } \\
\text { health sector) }\end{array}$ & $\begin{array}{l}\text { Measurement of costs } \\
\text { (physical units, valued } \\
\text { appropriately, all costs } \\
\text { included, itemised) }\end{array}$ & $\begin{array}{l}\text { QALY/DALY } \\
\text { weights clearly } \\
\text { defined }\end{array}$ & $\begin{array}{l}\text { Appropriate } \\
\text { conclusions } \\
\text { based on } \\
\text { results } \\
\end{array}$ & $\begin{array}{l}\text { Generalisability of } \\
\text { study to other } \\
\text { settings/ patient } \\
\text { groups discussed }\end{array}$ & $\begin{array}{l}\text { Conflict/s } \\
\text { of interest } \\
\text { disclaimed }\end{array}$ & $\begin{array}{l}\text { Ethical issues } \\
\text { discussed }\end{array}$ & $\begin{array}{l}\text { Risk analysis } \\
(++ \text { low risk, + } \\
\text { moderate } \\
\text { risk, - high risk) }\end{array}$ \\
\hline Amarasinghe [30] & $\begin{array}{l}\text { Uncertainty of compliance and } \\
\text { subsidy rates (10, 20, 25, 50, 75, } \\
\text { 100\%); N/A; Health Sector }\end{array}$ & $\checkmark$ & $\checkmark$ & $\checkmark$ & $x$ & $\checkmark$ & $x$ & + \\
\hline Cecchini [31] & $\begin{array}{l}\text { Simplified (sd }+15 \% \text { of mean, } \\
\text { max and min }+-60 \% \text { mean); } 3 \% \\
\text { (costs and effects); Health Sector }\end{array}$ & $x$ & $x$ & $\checkmark$ & $\checkmark$ & $\checkmark$ & $x$ & - \\
\hline Cobiac [32] & $\begin{array}{l}\text { Monte Carlo (2000 iterations) 95\% } \\
\mathrm{Cl} ; 3 \% \text { (costs and effects); Health Sector }\end{array}$ & $x$ & $\checkmark$ & $\checkmark$ & $x$ & $\checkmark$ & $x$ & - \\
\hline Cobiac [33] & $\begin{array}{l}\text { Monte Carlo, } 95 \% \text { Cl; } 3 \% \text { (costs } \\
\text { and effects); Health Sector }\end{array}$ & $x$ & $\checkmark$ & $\checkmark$ & $x$ & $\checkmark$ & $x$ & - \\
\hline Cobiac [34] & $\begin{array}{l}\text { Monte Carlo (2000 iterations), } \\
95 \% \mathrm{Cl} ; 3 \% \text { (costs and effects); } \\
\text { Societal and health sectors }\end{array}$ & $\checkmark$ & $\checkmark$ & $\checkmark$ & $x$ & $\checkmark$ & $x$ & + \\
\hline Cobiac [35] & $\begin{array}{l}\text { Monte Carlo, } 95 \% \mathrm{Cl} ; 3 \% \text { (costs } \\
\text { and effects); Health Sector }\end{array}$ & $\checkmark$ & $\checkmark$ & $\checkmark$ & $x$ & $\checkmark$ & $x$ & + \\
\hline Cobiac [36] & $\begin{array}{l}\text { Monte Carlo, } 95 \% \mathrm{Cl} \text {; } 3 \% \text { (costs } \\
\text { and effects); Health Sector }\end{array}$ & $\checkmark$ & $\checkmark$ & $\checkmark$ & $\checkmark$ & $\checkmark$ & $x$ & ++ \\
\hline Cobiac [37] & $\begin{array}{l}\text { Monte Carlo, } 95 \% \mathrm{Cl} ; 3 \% \text { (costs } \\
\text { and effects); Health Sector }\end{array}$ & $\checkmark$ & $\checkmark$ & $\checkmark$ & $\checkmark$ & $\checkmark$ & $\checkmark$ & ++ \\
\hline Dalziel [38] & $\begin{array}{l}\text { Uni-variate (effect size, cost, } \\
\text { utility, time horizon); } 5 \% \\
\text { (future costs): Societal }\end{array}$ & $x$ & $\checkmark$ & $\checkmark$ & $\checkmark$ & $x$ & $\checkmark$ & + \\
\hline Dalziel [39] & $\begin{array}{l}\text { One-way (1000 simulations); } \\
5 \% \text { (future costs); Health Sector }\end{array}$ & $x$ & $\checkmark$ & $\checkmark$ & $\checkmark$ & $x$ & $x$ & ++ \\
\hline Elley [40] & $\begin{array}{l}\text { Least squares regression model, } \\
95 \% \mathrm{Cl} ; 5 \% \text { (costs); Programme } \\
\text { Funder }\end{array}$ & $x$ & N/A & $x$ & $\checkmark$ & $x$ & $\checkmark$ & - \\
\hline Forster [41] & $\begin{array}{l}\text { Monte Carlo (2000 iterations), } \\
95 \% \text { Ul; 3\% (costs and effects); } \\
\text { Health Sector }\end{array}$ & $\checkmark$ & $\checkmark$ & $\checkmark$ & $\checkmark \checkmark$ & $\checkmark$ & $x$ & ++ \\
\hline $\mathbf{H a}[42]$ & $\begin{array}{l}\text { Monte Carlo (1000 iterations); 3\%; } \\
\text { Health Sector }\end{array}$ & $x$ & $x$ & $\checkmark$ & $\checkmark$ & $\checkmark$ & $x$ & - \\
\hline Higashi [43] & $\begin{array}{l}\text { Monte Carlo (2000 iterations), } 95 \% \\
\text { Cl; 3\% (costs and effects); Health Sector }\end{array}$ & $x$ & $x$ & $\checkmark$ & $\checkmark$ & $\checkmark$ & $x$ & - \\
\hline Higashi [44] & $\begin{array}{l}\text { Monte Carlo (2000 iterations), 95\% UI; } \\
3 \% \text { (costs and effects); Government } \\
\text { (implementation and maintenance) }\end{array}$ & $\checkmark$ & $x$ & $\checkmark$ & $\checkmark$ & $\checkmark$ & $x$ & + \\
\hline Jafar [45] & $\begin{array}{l}\text { Bayesian sensitivity (1000 repetitions), } \\
95 \% \text { Cl; } 5 \% \text { (costs and effects); Societal }\end{array}$ & $\checkmark$ & $x$ & $x$ & $\checkmark$ & $\checkmark$ & $\checkmark$ & + \\
\hline
\end{tabular}


Table 2 Assessing risk of bias in studies included in the review (Continued)

\begin{tabular}{|c|c|c|c|c|c|c|c|c|}
\hline Joo $[46]$ & No; N/A; Program Funder & $\checkmark$ & N/A & $x$ & $\checkmark$ & $x$ & $\checkmark$ & + \\
\hline Murray [47] & $\begin{array}{l}\text { Monte Carlo, multivariate uncertainty } \\
\text { analysis (range unclear); } 3 \% \text { (costs and } \\
\text { effects); Government (implementation } \\
\text { and health sector costs) }\end{array}$ & $x$ & $x$ & $\checkmark$ & $\checkmark$ & $\checkmark$ & $x$ & - \\
\hline Navarro [48] & $\begin{array}{l}\text { One-way (sensitivity analysis range } \\
\text { 39-59\%); N/A; Health Sector }\end{array}$ & $\checkmark$ & N/A & $\checkmark$ & $\checkmark$ & $\checkmark$ & $x$ & ++ \\
\hline Oldenburg [25] & Multivariate; N/A; Program Funder & $x$ & N/A & $\checkmark$ & $\checkmark$ & $x$ & $\checkmark$ & + \\
\hline Ortegon [49] & $\begin{array}{l}\text { One way, probabilistic uncertainty } \\
\text { analysis; } 3 \% \text { (costs and effects); Program } \\
\text { Funder and Health Sector }\end{array}$ & $x$ & $\checkmark$ & $x$ & $\checkmark$ & $\checkmark$ & $x$ & - \\
\hline Pritchard [28] & No; N/A; Program Funder & $x$ & N/A & $\checkmark$ & $\checkmark$ & $\checkmark$ & $\checkmark$ & ++ \\
\hline Ranson [29] & $\begin{array}{l}\text { Uncertainty around discount rates; 3\% } \\
\text { (costs and effects); Program Funder } \\
\text { (public sector) }\end{array}$ & $x$ & $\checkmark$ & $\checkmark$ & $\checkmark$ & $\checkmark$ & $x$ & + \\
\hline Sacks $[50]$ & $\begin{array}{l}\text { Monte Carlo (2000 iterations), 95\% } \\
\text { Cl; 3\% (costs and effects); Health } \\
\text { Sector (with some industry costs } \\
\text { included) }\end{array}$ & $x$ & $x$ & $\checkmark$ & $\checkmark$ & $\checkmark$ & $x$ & - \\
\hline Salkeld [27] & $\begin{array}{l}\text { One-way; } 5 \% \text { (costs and effects); } \\
\text { Health Sector (govt) }\end{array}$ & $\checkmark$ & $\checkmark$ & $\checkmark$ & $\checkmark$ & $x$ & $\checkmark$ & ++ \\
\hline Shearer [51] & $\begin{array}{l}\text { Multivariate (effectiveness, resource } \\
\text { use and costs); N/A; Program Funder } \\
\text { (govt.) }\end{array}$ & $x$ & N/A & $\checkmark$ & $\checkmark$ & $x$ & $x$ & - \\
\hline Snowdon [52] & $\begin{array}{l}\text { Monte Carlo (5000 iterations per country } \\
\text { model); probabilistic uncertainty analysis } \\
\text { of deaths averted; N/A: Govt. (cost offsets } \\
\text { excluded) }\end{array}$ & $x$ & N/A & $x$ & $\checkmark$ & $x$ & $x$ & - \\
\hline Zomer [53] & $\begin{array}{l}\text { Monte Carlo (1000 iterations) interquartile } \\
\text { range; Uncertainty based on compliance } \\
\text { levels; } 5 \% \text { (costs and effects): Health Sector }\end{array}$ & $x$ & $x$ & $x$ & $\checkmark$ & $\checkmark$ & $x$ & - \\
\hline
\end{tabular}

Abbreviations: Govt Government, Cl Confidence Interval, UI Uncertainty Interval, sd Standard Deviation. 
programme delivered by community health workers, a programme delivered by general practitioners, and a combination of the two, compared to no intervention. The interventions targeted diets, physical activity and smoking, and the primary outcomes were systolic blood pressure reduction and DALYs saved. Thus, some studies appear under multiple heads when assessing the impacts of interventions. Table 3 summarizes our main findings on economic evaluation.

\section{Tobacco control}

7 studies dealt with tobacco control, of which 5 were in low- and middle-income countries [42-45,49], 1 in a highincome country [51], and one in countries of all income levels [29]. Two studies [45,51] did not demonstrate costeffectiveness because their outcomes were other than QALYs or DALYs, namely the numbers of individuals who quit smoking and reduction in systolic BP [45,51]. 3 studies demonstrated interventions that were very cost-effective [42-44] with [43] investigating the cost-effectiveness of brief physician advice (general practitioner [GP] or other health professional) on tobacco cessation (1 min screening and 8 min advice session); [44] analysing excise tax increases (55\%-65\%, 55\%-75\%, 55\%-85\%), graphic warning labels on cigarette packs, mass media campaigns, smoking bans in public and smoking bans in the workplace; and [42] looking at a mass media campaign to reduce tobacco use. 2 studies assessed interventions that ranged from not cost-effective to very cost-effective depending on location and intervention type [29,49]. In study [29], compared to 'do nothing', a $10 \%$ price increase (55\%-65\%) in cigarettes was very cost-effective, and mass media interventions ranged from cost-effective to very costeffective (from \$25-510/DALY averted in East Asia \& Pacific and \$16-326/DALY averted in South Asia). In study [49] which used simulation modelling, increasing excise taxes on tobacco, bans on tobacco advertising, legislation and enforcement for clean indoor air in public places, and warning labels on cigarette packs were all very cost-effective in South and Southeast Asia.

\section{Alcohol reduction}

We found only 2 studies that evaluated cost-effectiveness of alcohol reduction primary prevention interventions $[32,48]$, both in Australia. Study [32] reported results ranging from not cost-effective (residential treatment for alcohol reduction) to very cost-effective (volumetric taxation, advertising bans, licensing controls on operating hours, brief primary prevention by GP, brief primary prevention by GP with telemarketing and support for GP recruitment to program, increased legal age of alcohol consumption from 18 to 21 years and mass media drink driving campaign). This study considered healthcare cost offsets in assessing cost-effectiveness. Study [48] was a cost-effectiveness analysis of an intervention consisting of screening by a GP and brief intervention if necessary (one appointment) among high-risk drinkers in a rural community in Australia. Outcomes were a reduction in high-risk drinking and the study estimated costs ranging from $\$ 175$-\$300 per high risk drinker moving to a low-risk status. However, no DALY or QALY outcomes were reported so a direct assessment in terms of the criteria of cost-effectiveness used in this review was not possible.

\section{Salt intake control}

We found 4 studies on salt control primary prevention interventions (Table 1), 2 in Australia [35,37], one in Vietnam [42], and one in a set of South East Asian countries [49]. In 2 studies, interventions ranged from being not cost-effective to cost-effective/very cost-effective depending on intervention type or setting $[35,49]$, and 2 studies $[37,42]$ found the interventions to be very costeffective. In study [35] incentives from government for moderate reduction in salt in processed foods and product labelling by manufacturers, and a government mandate for manufacturers to moderate salt limits in processed foods were cost-effective relative to 'doing nothing'; and individual dietary advice for those at increased/high risk of CVD was not cost-effective. In study [49], voluntary reduction in salt by the industry (15\%) was found to be very cost-effective; and a legislated $30 \%$ reduction in salt in industry also ranged from cost-effective to very costeffective, relative to doing nothing. In [37], compared to 'doing nothing', the mandatory reduction of salt in the manufacture of bread, cereals and margarines was found to be very cost-effective. In [42], a reduction in salt intake through voluntary manufacturer limits and a mass media campaign were very cost-effective.

\section{Physical activity/diet control}

Roughly three-fourths of the studies (20/28) examined diet and or physical activity-based primary preventions. Of these, $5[31,42,45,47,52]$ were in low- and middle-income countries. Of the 15 studies in high-income countries, 12 studies were conducted in Australia. The most common form of prevention evaluated was counselling (18 studies), although a few studies also evaluated legislation, taxation and mass media-based interventions. All 6 randomised controlled-trial (RCT)-embedded studies included in this review were in the physical activity/diet category, and of these only 1 was conducted in a middle-income country, Pakistan [45].

The studies in low- and middle-income countries covered a variety of primary prevention interventions. A study in Fiji and Tonga [52] examined the impact of tax interventions (lowering import duties on fruits and vegetables, raising import duties on fatty oils, removal of import duty benefits for processed meats), legislation (ban sales of 
Table 3 Cost-effectiveness of interventions

Study Currency Costs

$\$ 16$ million saved on $\mathrm{HD}$ treatment ICER (Incremental cost-effectiveness ratio)

Cost-effectiveness

Amarasinghe [30] 2003 AUD

When 100\% compliance, \$20 subsidy per

Very cost-effective

\$18 million saved on stroke treatment

GP visit \$810/DALY averted (all causes)

When 50\% compliance, \$25 subsidy rate,

$\$ 11,000 / D A L Y$ averted (all causes)

Cecchini [31] 2005 US\$

At 20 yrs, compared to control - Int1

China \$7785/DALY averted, India

\$6151/DALY averted; Int2 China

\$71/DALY averted, India \$952/DALY

averted; Int3 China \$7188/DALY

averted, India \$15552/DALY averted;

Int4 China cost-saving, India cost-saving

Cobiac [32] 2003 AUD

Cobiac [33] 2003 AUD

Cobiac [34] 2003 AUD

Cobiac [35]

2003 AUD

Cobiac [36]

2003 AUD

Cobiac [37]

2008 AUD

Compared with current best practice.

Int1 Dominant; Int2 Dominant; Int3

\$3300/DALY averted; Int4 \$6800/DALY \$3300/DALY averted; Int4 \$6800/DALY averted; Int5 \$10,000/DALY averted; Int6 $\$ 190,000 / D A L Y$ averted; Int7 Dominant; Int8 \$14000/DALY averted

Compared to 'do nothing'

Int1 \$130,000/DALY averted; Int2

$\$ 140,000 / D A L Y$ averted

Compared to 'do nothing' (medians)

Int1 Dominant; Int2 Dominant; Int3 \$3000/DALY averted; Int4 \$11,000/DALY averted; Int5 \$18,000/DALY averted; Int6 $\$ 79000 /$ DALY averted

Compared to 'do nothing'

Int1 Dominant; Int2 Dominant; Int3 \$260000-\$390000/DALY averted; Int4 160000-250000/DALY averted

Compared to 'do nothing', at $1 \mathrm{yr}$ (assumed 50\% decay in effectiveness after implementation):

Int1 Dominant; Int2 \$8600/DALY averted; Int3 \$12000/DALY averted; Int4 \$27000/DALY averted; Int5 \$280000/DALY averted; Int6 $\$ 950000 / D A L Y$ averted; Int7 $\$ 84000 /$ DALY averted

Compared to 'do nothing':

Int1 Dominant (Dominant to Dominant); Int2 \$44000/DALY averted (\$19000-\$100000/DALY averted); Int3 \$1000000/DALY
Int 1 Cost-effective in China, not cost-effective in India; Int 2 very cost-effective in China, cost-effective in India; Int 3 very cost-effective in China, not cost-effective in India; Int4 very cost-effective

Int1-5, 7-8 very cost-effective; Int 6 not cost-effective

Cost-effective

Int 1-5 very cost-effective; Int 6 cost-effective

Int 1 and 2 cost-effective; Int 3 not cost-effective Int4 unlikely to be cost-effective

Int 1-4 very cost-effective; Int 5 and 6 not cost-effective; Int7 cost-effective

Int 1 and 2 very cost-effective; Int 3 and 4 cost-effective; Int 5 not cost-effective 


$\begin{array}{ll}\text { Dalziel [38] } & 2003 \text { AUD } \\ \text { Dalziel [39] } & 2001 \text { NZ\$ }\end{array}$

Elley [40] $2001 \mathrm{NZ \$}$

Forster [41] 2003 AUD

$\mathrm{Ha}$ [42]

2007 VND

Higashi [43]

2006 VND

Higashi [44]

2006 VND

Jafar $[45]$

2007 US\$

Joo [46]
Int1 1945002/DALY averted; Int2 12324059/DALY averted; Int3 2416075/DALY averted: Int4 2211140/DALY

1742000/DALY averted

(I\$ 543/DALY averted)

physician advice

averted $(\$ 610000-2400000 /$ DALY

averted); Int4 \$1400000/DALY averted

(\$960000-2500000/DALY averted); Int5

$\$ 3200000 / D A L Y$ averted ( $\$ 1900000$

5900000/DALY averted)

Compared to control: \$46/QALY to

$\$ 19800 /$ ALY for all 8 interventions

Compared to control, at 40 year time point:

Very cost-effective

For intervention implemented 1 year,

effects lasting 4 years: \$2053/QALY gained:

Effects lasting 5 years: \$1663/QALY gained:

Effects lasting 10 years: \$1160/QALY gained

At 12 months, compared to control: \$1756

for 1 adult to move from a sedentary to

active state. Program cost $\$ 170.45$ per

patient per year

Compared to control: Int 1 \$12000/DALY

averted (cost saving -68000/DALY averted);

Int 2 \$13000/DALY averted (cost saving

-130000/DALY averted)

Compared to control: All interventions

Dominated

Compared to control: All pharmaceuticals

dominated by physician advice. pharmaceutical interventions not

cost-effective

Compared to control: All interventions dominate

Int1 8600VND/DALY averted (3400,

20100);Int2 4200VND/DALY averted (1700,

9900); Int3 2900VND/DALY averted (1100,

6700); Int4 500VND/DALY averted (300,

1200); Int5 78300VND/DALY averted (43700

176300): Int6 67900VND/DALY averted

(28200-332000); Int7 336800VND/DALY

averted (169300, 822900)

Compared to no intervention:

Int $1 \$ 23 / \mathrm{mmHg}(\$ 7-\$ 101 / \mathrm{mmHg})$;

int 2 Dominated (dominated to

$730 / \mathrm{mmHg}$ ); Int $3 \$ 206 / \mathrm{mmH}$

(Dominated to $\$ 807 / \mathrm{mmHg}$ )

Inconclusive

Very cost-effective

Very cost-effective

Inconclusive

Public health int $\$ 976 /$ person

to reach target weight; Remote

int. \$1637/person to reach target

Inconclusive 
Table 3 Cost-effectiveness of interventions (Continued)

\begin{tabular}{|c|c|c|}
\hline Murray [47] & $1 \$ 2000$ & $\begin{array}{l}\text { Int } 1 \text { \$37/DALY averted; Int2\$19/DALY } \\
\text { averted; Int3 \$14/DALY averted; Int4 } \\
\text { \$17/DALY averted (personal interventions } \\
\text { \$36-90/DALY) }\end{array}$ \\
\hline Navarro [48] & 2005-2006 AUD & \\
\hline Oldenburg [25] & 1990 AUD & $\begin{array}{l}\text { Int3 (only intervention which reached } \\
\text { the maintenance stage of behavioural } \\
\text { intervention) } \$ 22.06 / \text { unit of CVD risk } \\
\text { reduction }\end{array}$ \\
\hline
\end{tabular}

Int 2 compared to Int3 \$14/DALY

averted; (Int 2 to Int 4) compared

Compared to 'do nothing': 10\%

increase in screening rate $\$ 217 /$ risky

drinker reducing alcohol consumption;

$20 \%$ increase $\$ 205 ; 100 \%$ increase $\$ 216$

Ortegon [49] $\quad 1 \$ 2005$

Compared to no intervention: Int1 \$116/DALY averted; Int2 \$87/DALY averted; Int3 \$187/DALY averted; Int4 \$162/DALY averted; Int5 \$195/DALY averted; Int6 \$958/DALY averted; Int7 \$1179/DALY averted; Int8 \$197/DALY averted; Int9 \$901991/DALY averted; Int10 \$191/DALY averted

Compared to control: Int 1 \$9.76/extra kg lost (12\% reduction in BP); Int 2

$\$ 7.30 /$ extra $\mathrm{kg}$ lost (7\% reduction in BP)

Compared to control: Int 1: East Asia \& Pacific \$2-50/DALY averted, South Asia \$1-33/DALY averted; Int 2: East Asia \& Pacific \$25-510/DALY averted, South Asia \$16-326/DALY averted

Compared to 'do nothing': Int 1 Dominant (\$30/DALY averted, 95\% Cl 20-40); Int 2

Dominant (\$1800/DALY averted, 95\%

Cl 1360-2170)

Compared to routine care, both interventions not cost-effective or effective (no significant change in risk): Except for Int 1 for high risk

males \$39440/LYS and \$29574/QALY

Very cost-effective

cost-efertive; Int6 not in Bangladesh, Dem Rep Kort indi, very cost-effective, Bem Rep Korea, India, very cost-effective in Myanmar or Nepal, cost-effective in Bangladesh, India, Dem Rep Korea, very cost-effective in Bhutan and Maldives; Int9 not cost-effective

Inconclusive

Int 1 Very cost-effective; Int 2 cost-effective to very cost-effective

Very cost-effective

Int 1 for high risk males very cost-effective

Brief advice: \$1910/quitter (\$1273-3820); Telephone counselling: \$606/quitter $(\$ 505-757)$

Snowdon [52] 2006 Fiji dollar (FJD)

At 1 year Most effective: Tonga Ban on sale of all fatty meats TOP

30974/6.61 deaths averted; Fiji -

cool storage available at all markets

2006 Tongan Pa'anga (TOP) 
Table 3 Cost-effectiveness of interventions (Continued)

FJD1600149/65.54 deaths averted;
Lowest costs: Tonga - removal of
licensing requirements for roadside
vendors selling local produce
TOP0/death averted; Fiji- import
duty (15\%) added to all oils
FJD396/17.43 deaths averted

Zomer [53] 2012 AUD 100\% compliance: $\$ 50,000 / L Y S$

Very cost-effective

NB. All studies are cost-effectiveness analyses. Abbreviations: CEA - Cost-Effectiveness Analysis; FJD - Fijian Dollar; TOP - Tongan Pa-anga; AUD - Australian Dollar; DALY - Disability-Adjusted Life Years; QALY -

Quality-Adjusted Life Years; LYS - Life Years Saved; Cl - Confidence Interval; USD - United States Dollar; I\$ - international dollars; VND - Vietnamese Dong; mmHg - millimetres of mercury; NZ\$ - New Zealand Dollar;

Int - Intervention; yrs - years; ICER - Incremental Cost-Effectiveness Ratio. 
fatty meats and fatty processed foods) and enhancing cool storage for fruits, vegetables and fish, concluding that tax policies and legislation were likely to be much more costeffective (in terms of deaths averted) than expansion of storage facilities. However, outcomes were not expressed in DALYs or QALYs. The study for Pakistan [45] was also difficult to assess from a cost-effectiveness perspective as the outcome was systolic BP. Study [48] used mathematical modelling together with effectiveness data from mostly high-income countries to assess the costeffectiveness of 7 programmes directed to diets and physical activity in 6 middle-income countries, of which 2 (China and India) were in the Asia-Pacific region. The programs evaluated were school-based primary preventions, worksite primary preventions, mass media campaigns, tax policies (affecting prices for fruits, vegetables and foods rich in fat), GP counselling, regulation of food advertising and food labelling. Regulation of food advertising, food labelling and tax policies ranged from cost-effective to very cost-effective in both China and India. In China, moreover, worksite primary preventive interventions and mass media campaigns were assessed to be very cost-effective over a long (50-year) horizon. However, other interventions were not cost-effective in either country. In Vietnam, a mass media campaign aimed at reducing cholesterol [42] was assessed to be very costeffective, although other than salt intake it was not clear if any other lifestyle factors were targeted by the intervention. A mass media campaign targeting BP and cholesterol control was also found to be very cost-effective in [47] in Southeast Asian countries.

In studies conducted in high income countries (Australia, New Zealand and South Korea), 5 either did not explicitly assess cost-effectiveness or used outcome measures that were not expressed in DALYs or QALYs $[25,28,40,46,53]$. Study [46], set in Korea, concluded that it was more cost-effective to educate individuals about dietary and lifestyle changes to lower weight than to do so remotely via the internet; study [40] set in New Zealand concluded that GP advice and prescription for physical activity with telephone follow-up by an exercise specialist increased physical activity among 40-79 year olds at a 'reasonable cost'; study [28] set in Australia concluded that nutritional counselling to overweight or hypertensive patients lowered weight at a rate of $\$ 7-\$ 10$ of programme costs per lost kilogram; another study [25] concluded that behavioural counselling was more costeffective in lowering CVD risk factors than strategies focused on risk-factor education among individuals or behavioural counselling plus financial incentives; and study [53] also set in Australia, found an intervention promoting dark chocolate consumption was very cost-effective.

The remaining 10 studies were set in Australia (1 in New Zealand). Of these, a significant number focused on counselling, with and without GPs, and these interventions were generally cost-effective. In study [30], GP advice for sufficient physical activity upon presentation at a clinic was compared to no advice and found to be costeffective/very cost-effective depending on patient compliance rates. In study [27] GPs were provided with a video and an instructional guide to assess risk factors and to plan risk factor behaviour change and patients also received a video on lifestyle behaviours and risk factor assessment. In addition, a small group received a self-help booklet. Video-based interventions were cost-effective or very cost-effective for high-risk males, but were not costeffective for other population subsets. In study [37], dietary advice from doctor or dietician or referral to more intensive lifestyle program with specialised counselling (for those with $\geq 15 \%$ risk of CVD) were found to be costeffective compared to doing nothing. In study [39] a physical activity counselling program in New Zealand implemented by a GP or nurse with telephone follow-up was found to be very cost-effective compared to existing practice. In study [33], group counselling for changing physical activity levels and nutritional patterns, and a low-calorie diet with physical activity advice were found to be cost-effective compared to no intervention although health gains were overall quite low. On the other hand [34] found GP physical activity prescription programs to be very cost-effective and GP referral to an exercise physiologist to be cost-effective. Only one study [36] found individual and group counselling to be not costeffective. Study [36] also assessed community-based primary prevention including sponsorship and promotion for fruit and vegetable consumption, information mail outs, all of which were very cost-effective.

Among other primary prevention interventions, study [50] found junk food taxes and food labelling targeted at Australia's adult population to be individually very costeffective. Study [34] assessed interventions for increasing physical activity, finding the use of pedometers, a mass media campaign, internet advertising and a program to encourage the use of active transport to be very costeffective; study [38] examined 8 diet programs and found all to be very cost-effective; and finally, in study [41], a hypertension diet combined with exercise and a low-fat diet were compared to no intervention and both were very cost-effective, although the overall health benefits were small.

Several of these studies addressed BMI as a cardiovascular risk factor. Despite BMI by itself not being a reliable predictor of CVD, in the absence of other measures such as systolic BP and diabetes, it offers some indication that other risk factors for CVD may be present, as determined in a recent, comprehensive Lancet study [55]. Weight loss studies included in this review follow this line with weight gain considered an intermediate risk factor for CVD with 
studies $[27,33,34,37,39,41,52]$ modelling QALYs or DALYs based on, for example, the relative risk of cardiovascular (and other) disease when at a particular weight. Studies $[28,40,46]$ use weight loss as the direct outcome.

\section{Discussion}

We find first that multiple tobacco control interventions, particularly taxes on tobacco, advertising restrictions, warning labels, mass media campaigns and GP consultations were cost-effective to very cost-effective. Second, available studies show that alcohol taxation, advertising restrictions, mass media campaigns against drunk driving and GP-based primary preventions range from costeffective to very cost-effective, although in this case, the studies are limited to Australia. Third, voluntary manufacturer limits, or mandated or incentivized reduction in salt in processed foods were very cost-effective. However, dietary advice on salt intake for individuals at high risk of CVD was not cost-effective. Fourth, regulation of food advertising, food labelling and taxes on fatty foods/oils are very cost-effective, although the evidence on worksite primary prevention was unclear. Studies, primarily from developed countries in the region, also show that dietary and physical activity counselling through health personnel (with some follow up) is cost-effective. Community-based primary prevention on diet and physical activity were also found to be cost-effective but their benefits were small.

Unfortunately we also find a serious shortage of information on cost-effectiveness for primary lifestyle prevention related to CVD in the Asia-Pacific region. Only 28 studies met our inclusion criteria, covering various lifestyle and behavioural interventions for primary prevention of CVD. A large number of studies analysed in this review were conducted in developed countries of the region, mainly Australia. Only 8 studies were conducted exclusively in low- or middle-income countries of the Asia Pacific Region (Fiji and Tonga; China and India; Pakistan; Vietnam) so that generalizing our findings on costeffectiveness of primary prevention interventions to the broader region is difficult. Only one study covered a broad cross-section of countries in the region, low- and highincome, and was limited to tobacco control. And a number of studies did not use DALYs or QALYs as outcomes.

Overall 20/28 of the studies demonstrated costeffectiveness of an intervention, but we cannot rule out publication bias skewing our results towards including studies demonstrating cost-effectiveness. However, the share of studies reporting (cost) effectiveness is similar to comparable reviews in the field [56].

With the exception of $[40,41]$ studies included in this review generally rely on short-term effects of interventions to construct cost-effectiveness estimates. Short-term effects can be misleading if there is relapse in behaviour, as in interventions targeting weight loss or smoking or, when intervention is at the population level, there are saturation effects [57]. In particular, mathematical modelling the effects of an intervention over the lifetime of an individual are susceptible to this problem given their estimates of economic impacts rely on modelling of behavioural change beyond the intervention period [27,33,38-44,49]. In addition, RCT-based analyses sometimes rely on conditions that are unlikely to be replicated in other real life settings. Sensitivity analyses can address this to an extent and many of the studies included in this review do include analyses that allow for variation in the estimates of program effectiveness. The sensitivity analyses took various forms, such as allowing for decay in behavioural interventions over time or variations in compliance rates, or in some cases, varying rates of program effectiveness. However, it is difficult to evaluate how effective these sensitivity analyses were in capturing uncertainty about the full range of outcomes that were possible and/or long-term program effects. For those studies which were modelled for over a year, discount rates were similar, with most authors discounting both costs and effects by 3 to 5 percent annually. Another limitation is that several studies did not provide a clear definition of the DALY or QALY weighting used in their analysis $[31,42-45,47,50,53]$.

Primary prevention interventions also varied considerably in the studies considered. Interventions associated with tobacco control, alcohol reduction, physical activity/ diet control and salt control can broadly be divided into counselling, legislation and mass media. Yet, in their implementation, the interventions evaluated varied by the age and occupational group of the population targeted, gender, risk level for CVD; the length of intervention; or the implementing body be it general practitioners, legislators or researchers. Thus, for counselling-mediated physical activity and diet primary prevention, the target population varied from the whole population [47], to those 20 years or older who were also overweight or obese [41] to those who were 18 years or older and ambulance officers [25].

In consequence it is difficult to aggregate the costeffectiveness findings of individual studies, say through meta-analysis based on results from high quality studies. Much of the evidence for effectiveness, even when applied to low- and middle-income countries in the region, has come from high-income countries, some from outside the Asia-Pacific. The cost data, derived from the WHOCHOICE model in some of the studies, conforms to a standardized approach that may not be suited to the considerable heterogeneity across health systems in the region. Moreover the assessment of costs was variable, with some studies limited to costing the primary prevention alone, others to taking account of the costs of healthcare use associated with the primary prevention and a few also including household costs. 
Finally, the review makes clear the need to have graded cost-effectiveness data for multiple interventions and standardized methods for estimating costs and outcomes for individual countries in the region to guide policy choices. Australia is the only country of the Asia-Pacific Region with a developed evidence base and a relative standardisation of methods [32-37], and could serve as a benchmark for similar studies elsewhere in the region.

\section{Conclusion}

Although there is a large international literature on the effectiveness and cost-effectiveness of primary prevention interventions targeting lifestyle factors for CVD, a closer examination points to a very limited literature in the AsiaPacific Region. Even this limited literature is characterized by variations in the interventions evaluated across countries and use of dissimilar methodologies, and has its major focus on high-income countries in the region. This constitutes a major gap in the literature at a time when NCDs are acquiring increasing significance in the region. As countries turn towards universal healthcare coverage, policymakers will need high quality advice about efficient strategies regarding NCDs, particularly those focusing on prevention, to avoid burdening health systems with large numbers of individuals with chronic conditions that are expensive to treat.

\section{Additional files}

Additional file 1: Full list of sources and search strategy.

Additional file 2: Eligibility checklist.

Additional file 3: Data extraction and management.

Additional file 4: Assessment of risk of bias in included studies.

\begin{abstract}
Abbreviations
CVD: Cardiovascular disease; APR: Asia-Pacific Region; DALY: Disability-adjusted life years; RR: Risk ratio; GDP: Gross domestic product; MONICA: Monitoring trends and determinants in cardiovascular disease; CEA: Cost-effectiveness analyses; QALY: Quality-adjusted life years; CE: Cost effective; VCE: Very cost-effective; NCE: Not cost effective; Cl: Confidence interval; COPD: Chronic obstructive pulmonary disease; UI: Uncertainty interval; sd: Standard deviation; GP: General practitioner; SEARO: South-East Asia Regional Office; RCT: Randomised control trial; BP: Blood pressure; BMI: Body mass index; CHOICE: Choosing interventions that are cost effective; NRT: Nicotine replacement therapy; HHE: Home health education; Govt: Government; Int: Intervention; ICER: Incremental cost-effectiveness ratio; LYS: Life-years saved; FJD: Fijian dollar; TOP: Tongan pa-anga; AUD: Australian dollar; USD: United States dollar; VND: Vietnamese dong;
\end{abstract} mmHg: Millimetres of mercury; NZ\$: New Zealand dollar.

Competing interests

The authors declare that they have no competing interests.

\section{Authors' contributions}

LS and AM conceived the study with inputs from AK. LS, AM and AK performed the review of literature and contributed in analysis. LS and AM wrote the initial draft of the manuscript and AK contributed to the draft. All authors read and approved the final manuscript.

\section{Acknowledgement}

Anup Karan is supported by the Wellcome Trust Capacity Strengthening Strategic Award to the Public Health Foundation of India and a consortium of UK universities. Ajay Mahal is supported by Establishment Grant funded by the Alan and Elizabeth Finkel Foundation at the School of Public Health and Preventive Medicine at Monash University. The funders had no role in study design, data analysis, decision to publish, or preparation of the manuscript. The study was approved by the review panel of Monash University.

\section{Author details}

${ }^{1}$ School of Public Health and Preventive Medicine, Monash University, Alfred Centre, 99 Commercial Road, Melbourne, VIC 3004, Australia. Indian Institute of Public Health Gandhinagar (IIPHG), Sardar Patel Institute Campus, Thaltej, Ahmedabad 380 054, India. ${ }^{3}$ Nuffield Department of Population Health, University of Oxford, Oxford, UK.

Received: 9 May 2014 Accepted: 3 November 2014

Published online: 19 November 2014

\section{References}

1. Institute for Health Metrics and Evaluation (IHME): Global Burden of Disease Study 2010 (GBD 2010). [http://ghdx.healthdata.org/]

2. Reddy KS: Cardiovascular diseases in the developing countries: dimensions, determinants, dynamics and directions for public health action. Public Health Nutr 2006, 5(1a):231-237.

3. World Health Organization: Global Burden of Disease Database. [http://www. who.int/healthinfo/global_burden_disease/en/]

4. World Bank: World Data Bank - The World Bank World Development Indicators. [http://databank.worldbank.org/data/views/variableSelection/selectvariables. aspx?source=world-development-indicators]

5. Yang L, Sung HY, Mao Z, Hu T, Rao K: Economic costs attributable to smoking in China: update and an 8-year comparison, 2000-2008. Tob Control 2011, 20(4):266-272.

6. Iqbal MP: Economic development by reducing the burden of cardiovascular disease in South Asia. Pakistan J Med Sci 2012, 28(3):4-566.

7. Karan A, Engelgau M, Mahal A: The household level economic burden of heart disease in India. Trop Med Int Health 2014. doi:10.1111/tmi.12281.

8. Alwan A, Armstrong T, Bettcher D, Boerma T, Branca F, Ho JCY, Mathers C, Martinez R, Poznyak V, Roglic G, Riley L, D'Espaignet E, Stevens G, Taubert K, Xuereb G: Global Atlas on Cardiovascular Disease Prevention and Control. Geneva: World Health Organization, World Heart Federation and World Stroke Organization; 2011.

9. Dans A, Ng N, Varghese C, Tai ES, Firestone R, Bonita R: The rise of chronic non-communicable diseases in southeast Asia: time for action. Lancet 2011, 377:680-689.

10. Govindaraju DR, Cupples LA, Kannel WB, O'Donnell CJ, Atwood LD, D'Agostino RB, Fox CS, Larson M, Levy D, Murabito J, Vasan RS, Splansky GL, Wolf AP, Benjamin EJ: Genetics of the Framingham Heart Study population. Adv Genet 2008, 62(8):33-65.

11. Jousilahti P, Vartiainen E, Tuomilehto J, Puska P: Sex, age, cardiovascular risk factors, and coronary heart disease: a prospective follow-up study of 14786 Middle-Aged Men and Women in Finland. Circulation 1999, 99:1165-1172

12. Kuulasmaa K, Tunstall-Pedoe H, Dobson A, Fortmann S, Sans S, Tolonen H, Evans A, Ferrario M, Tuomilehto J: Estimation of contribution of changes in classic risk factors to trends in coronary-event rates across the WHO MONICA Project populations. Lancet 2000, 355:675-687.

13. Kristiansen IS, Eggen AE, Thelle DS: Cost effectiveness of incremental programmes for lowering serum cholesterol concentration: is individual intervention worth while? BMJ 1991, 302:1119-1122

14. Gaziano TA, Galea G, Reddy KS: Scaling up interventions for chronic disease prevention: the evidence. Lancet 2007, 370(9603):1939-1946

15. World Health Organization: Package of Essential Noncommunicable (PEN) Disease Interventions for Primary Health Care in Low-Resource Settings. ; 2010

16. The World Bank: Data - How we classify countries. [http://data.worldbank org/about/country-and-lending-groups]

17. Asaria P, Chisholm D, Mathers C, Ezzati M, Beaglehole R: Chronic disease prevention: health effects and financial costs of strategies to reduce salt intake and control tobacco use. Lancet 2007, 370(9604):2044-2053.

18. Shroufi A, Chowdhury R, Anchala R, Stevens S, Blanco P, Han T, Niessen L, Franco $\mathrm{OH}$ : Cost effective interventions for the prevention of 
cardiovascular disease in low and middle income countries: a systematic review. BMC Public Health 2013, 13(285):1-13.

19. Department of Immigration and Border Protection, Australian Government, Historical Migration Statistics, Canberra. 2014. [https://www. immi.gov.au/media/statistics/historical-migration-stats.htm]

20. World Health Organization: Asia Pacific Observatory on Health Systems and Policies. [http://www.wpro.who.int/asia_pacific_observatory/en/]

21. Chapman S, Wakefield M: Tobacco control advocacy in Australia: reflections on 30 years of progress. Heal Educ Behav 2001, 28(3):274-289.

22. Popkin BM: The nutrition transition and obesity in the developing world. J Nutr 2001, 131(suppl):871-873

23. Evers $\mathrm{S}$, Goossens $M$, de Vet $H$, van Tulder M, Ament A: Criteria list for assessment of methodological quality of economic evaluations: consensus on Health Economic Criteria. Int J Technol Assess Heal Care Technol Assess 2007, 11:22.

24. Centre for Public Health Excellence: Methods for development of NICE public health guidance. London: National Institute for Health and Clinical Excellence; 2006

25. Oldenburg B, Owen N, Parle M, Gomel M: An economic evaluation of fou work site based cardiovascular risk factor interventions. Heal Educ Behav 1995, 22(1):9-19.

26. The World Bank Databank: 2013, [http://databank.worldbank.org/data]

27. Salkeld G, Phongsavan P, Oldenburg B, Convery P, Graham-clarke P, Walker S, Shaw J: The cost-effectiveness of a cardiovascular risk reduction program in general practice. Health Pol (New York) 1997, 41:105-119.

28. Pritchard DA, Hyndman J, Taba F: Nutritional counselling in general practice : a cost effective analysis. J Epidemiol Community Health 1999, 53:311-316

29. Ranson MK, Jha P, Chaloupka FJ, Nguyen SN: Global and regional estimates of the effectiveness and cost-effectiveness of price increases and other tobacco control policies. Nicotine Tob Res 2002, 4:311-319.

30. Amarasinghe AK: Cost-effectiveness implications of GP intervention to promote physical activity : evidence from Perth. Cost Eff Resour Alloc 2010, 8(10):1-6.

31. Cecchini M, Sassi F, Lauer JA, Lee YY, Guajardo-barron V, Chisholm D: Tackling of unhealthy diets, physical inactivity, and obesity : health effects and cost-effectiveness. Lancet 2010, 376(9754):1775-1784

32. Cobiac L, Vos T, Doran C, Wallace A: Cost-effectiveness of interventions to prevent alcohol-related disease and injury in Australia. Addiction 2009, 104:1646-1655.

33. Cobiac L, Vos T, Veerman L: Cost-effectiveness of weight watchers and the lighten up to a healthy lifestyle program. Aust $N Z$ J Public Health 2010, 34(3):240-247.

34. Cobiac LJ, Vos T, Barendregt JJ: Cost-effectiveness of interventions to promote physical activity: a modelling study. PLoS Med 2009, 6(7):e1000110.

35. Cobiac $L$, Vos $T$, Veerman $J$ : Cost-effectiveness of interventions to reduce dietary salt intake. Heart 2010, 96:1920-1925.

36. Cobiac LJ, Vos T, Veerman JL: Cost-effectiveness of interventions to promote fruit and vegetable consumption. PLOS One 2010, 5(11):e14148.

37. Cobiac LJ, Magnus A, Lim S, Barendregt JJ, Carter R, Vos T: Which interventions offer best value for money in primary prevention of cardiovascular disease? PLoS One 2012, 7(7):1-10.

38. Dalziel K, Segal L: Time to give nutrition interventions a higher profile: cost-effectiveness of 10 nutrition interventions. Health Promot Int 2007 22(4):271-283

39. Dalziel K, Segal L, Elley CR: Cost utility analysis of physical activity counselling in general practice. Aust N Z J Public Health 2006, 30(1):57-63.

40. Elley R, Kerse N, Arroll B, Swinburn B, Ashton T: Cost-effectiveness of physical activity counselling in general practice. N Z Med J 2004 117(1207):1-15.

41. Forster M, Veerman JL, Barendregt JJ, Vos T: Cost-effectiveness of diet and exercise interventions to reduce overweight and obesity. Int J Obes 2011, 35(8):1071-1078

42. Ha DA, Chisholm D: Cost-effectiveness analysis of interventions to prevent cardiovascular disease in Vietnam. Health Policy Plan 2011 26(3):210-222

43. Higashi $\mathrm{H}$, Barendregt JJ: Cost-effectiveness of tobacco control policies in Vietnam: the case of personal smoking cessation support. Addiction 2012, 107(3):658-670.
44. Higashi H, Truong KD, Barendregt JJ, Nguyen PK, Vuong ML, Nguyen TT, Hoang PT, Wallace AL, Tran TV, Le CQ, Doran CM: Cost effectiveness of tobacco control policies in Vietnam. Appl Health Econ Health Policy 2011, 9(3):183-196.

45. Jafar TH, Islam M, Bux R, Poulter N, Hatcher J, Chaturvedi N, Ebrahim S, Cosgrove P: Cost-effectiveness of community-based strategies for blood pressure control in a low-income developing country: findings from a cluster-randomized, factorial-controlled trial. Circulation 2011, 124(15):1615-1625.

46. Joo N, Park Y, Park K, Kim C, Kim B: Cost-effectiveness of a communitybased obesity control programme. J Telemed 2010, 16:63-67.

47. Murray CJL, Lauer JA, Hutubessy RCW, Niessen L, Tomijima N, Rodgers A, Lawes CMM, Evans DB: Effectiveness and costs of interventions to lower systolic blood pressure and cholesterol: a global and regional analysis on reduction of cardiovascular-disease risk. Lancet 2003, 361(9359):717-725.

48. Navarro HJ, Shakeshaft A, Doran CM, Petrie DJ: The potential costeffectiveness of general practitioner delivered brief intervention for alcohol misuse: evidence from rural Australia. Addict Behav 2011, 36(12):1191-1198.

49. Ortegon M, Lim S, Chisholm D, Mendis S: Cost effectiveness of strategies to combat cardiovascular disease, diabetes, and tobacco use in sub-Saharan Africa and South East Asia : mathematical modelling study. BMJ 2012, 344:1-15.

50. Sacks G, Veerman JL, Moodie M, Swinburn B: 'Traffic-light' nutrition labelling and 'junk-food' tax: a modelled comparison of cost-effectiveness for obesity prevention. Int J Obes (Lond) 2011, 35(7):1001-1009.

51. Shearer J, Shanahan $M$ : Cost effectiveness analysis of smoking cessation interventions. Aust N Z J Public Health 2006, 30(5):428-434.

52. Snowdon W, Moodie M, Schultz J, Swinburn B: Modelling of potential food policy interventions in Fiji and Tonga and their impacts on noncommunicable disease mortality. Food Policy 2011, 36(5):597-605.

53. Zomer E, Owen A, Magliano DJ, Liew D, Reid CM: The effectiveness and cost effectiveness of dark chocolate consumption as prevention therapy in people at high risk of cardiovascular disease : best case scenario analysis using a Markov model. BMJ Open 2012, 344:1-9.

54. Prochaska JO: Transtheoretical Model of Behavior Change. In Encyclopedia of Behavioral Medicine. Edited by Gellman MD, Turner JR. New York: Springer; 2013:1997-2000.

55. Wormser D, Kaptoge S, Di Angelantonio E, Wood AM, Pennells L, Thompson A: Separate and combined associations of body-mass index and abdominal adiposity with cardiovascular disease: collaborative analysis of 58 prospective studies. Lancet 2011, 377(9771):1085-1095.

56. Dutton $\mathrm{GR}$, Laitner MH, Perri MG: Lifestyle interventions for cardiovascular disease risk reduction: a systematic review of the effects of diet composition, food provision, and treatment modality on weight loss. Curr Atheroscler Rep 2014, 16(10):442-456.

57. Elfhag K, Rössner S: Who succeeds in maintaining weight loss? A conceptual review of factors associated with weight loss maintenance and weight regain. Obes Rev 2005, 6(1):67-85.

\section{doi:10.1186/s12992-014-0079-3}

Cite this article as: Sutton et al: Evidence for cost-effectiveness of lifestyle primary preventions for cardiovascular disease in the Asia-Pacific Region: a systematic review. Globalization and Health 2014 10:79.

\section{Submit your next manuscript to BioMed Central and take full advantage of:}

- Convenient online submission

- Thorough peer review

- No space constraints or color figure charges

- Immediate publication on acceptance

- Inclusion in PubMed, CAS, Scopus and Google Scholar

- Research which is freely available for redistribution 